\title{
EL SISTEMA DE FINITO EN LA CLÁUSULA DEL BRIBRI DE COROMA SEGÚN LA GRAMÁTICA SISTÉMICO-FUNCIONAL
}

\author{
Sara Pacchiarotti
}

\begin{abstract}
RESUMEN
Este artículo analiza los subsistemas que componen el sistema de FINITO en bribri en el marco de la gramática sistémico-funcional de M.A.K. Halliday. A partir de la descripción de cada uno de los subsistemas se intenta, al final, construir un posible sistema de FINITO para esta lengua. También se aborda la cuestión de la transitividad disminuida en cláusulas perfectivas negativas desde un punto de vista sistémico-funcional.

Palabras clave: bribri, gramática sistémico-funcional, sistema de FINITO, sistema de MODALIDAD, polaridad negativa en cláusulas perfectivas.
\end{abstract}

\begin{abstract}
This article seeks to analyze the subsystems that constitute the system of FINITENESS in Bribri in the frame of systemic-functional grammar of M.A.K. Halliday. Starting from the description of each subsystem, the author seeks to build a possible system of FINITENESS for the Bribri language. The issue of diminished transitivity in perfective negative clauses is also addressed from a systemic-functional perspective.

Key words: bribri, systemic-functional grammar, system of FINITENESS, system of MODALITY, negative polarity in perfective clauses.
\end{abstract}

\section{La lengua bribri y sus hablantes}

El bribri es una lengua perteneciente a la estirpe chibchense (Constenla 1991), familia que se extiende desde la costa oriental de Honduras hasta la región central de Colombia. De las lenguas que la conforman, el guatuso, el bribri, el cabécar, el boruca y el térraba se hablan en Costa Rica. El bribri y el cabécar, además, conforman el grupo viceíta.

ML. Sara Pacchiarotti. Departamento de Lingüística, Universidad de Oregon.

Correo electrónico: sara.pacchiarotti001@ucr.ac.cr

Recepción: 24- 04- 2012

Aceptación: 10- 07- 2012 
Los bribris habitan la región sureste de Costa Rica, particularmente ambos lados de la Cordillera de Talamanca (Jara Murillo 2004a: 90). Del lado del Océano Pacífico, los bribris se ubican en el cantón de Buenos Aires, provincia de Puntarenas, sobre todo en las reservas de Salitre y Cabagra. Del lado del Océano Atlántico, los bribris viven en la Reserva de Talamanca, en el cantón de Talamanca, en la provincia de Limón. Además, se distinguen tres variedades dialectales: Amubre (Talamanca oriental), Coroma (Talamanca occidental) y Salitre (Pacífico Sur). Por lo que atañe al presente artículo, el material de análisis pertenece exclusivamente al dialecto de Coroma.

\section{El Finito en la gramática sistémico-funcional}

El elemento del Modo² está formado por dos partes: el Sujeto, que es generalmente un grupo nominal; y el operador Finito, que es parte de un grupo verbal. El elemento Finito es uno de los operadores verbales que puede expresar pasado o modalidad $\mathrm{y}$, dependiendo de la lengua, puede estar fusionado con el verbo léxico. La secuencia Sujeto + Finito sirve para realizar la selección de modo en la cláusula. El resto de la cláusula se define, en la gramática sistémico-funcional, como Residuo.

Desde el punto de vista paradigmático, el principio general que rige la expresión del sistema del MODO en la cláusula puede resumirse de la siguiente manera: la categoría gramatical utilizada generalmente para intercambiar información es el indicativo. Dentro de la categoría del indicativo, la expresión característica de una declaración es la declarativa, mientras que la expresión de una pregunta es la interrogativa. La función semántica del elemento Modo es la de llevar la carga de la cláusula como evento interactivo. A su vez, los elementos que lo componen tienen una función semántica específica, como se describirá en los siguientes apartados.

El elemento Finito tiene la función de circunscribir la proposición, es decir, al convertirla en finita, relaciona la proposición con su contexto en el evento de habla. Esta función puede ser llevada a cabo en uno de dos modos: (i) mediante la referencia al tiempo de la enunciación; y (ii) mediante la referencia al juicio del hablante. En términos gramaticales, (i) se define como tiempo primario, mientras que (ii) se define como modalidad.

El tiempo primario significa pasado, presente o futuro, en el momento de la enunciación: es el tiempo relativo al 'ahora'. Una proposición (mas no una propuesta) se hace discutible a través de su ubicación en un determinado tiempo en relación con el evento de habla.

La modalidad se traduce en posibilidad o imposibilidad en el caso de una proposición, o bien, deseabilidad o indeseabilidad en el caso de una propuesta. Una proposición o una propuesta llegan a ser discutibles si se evalúan en términos del grado de probabilidad u obligatoriedad que se les asocia.

Tanto la modalidad como el tiempo primario son manifestaciones de la deixis interpersonal: localizan el intercambio en el espacio semántico que se abre entre el hablante y el oyente. El tiempo primario interpreta el tiempo de forma interpersonal, definiendo el 'presente' para el hablante y el oyente en el momento de la enunciación. Asimismo, la modalidad establece un área de incertidumbre en la que el hablante puede expresar, o pedirle al oyente que lo haga, un juicio sobre la validez de lo que se está diciendo. El elemento Finito se expresa, por ende, mediante un operador verbal que puede ser modal o temporal.

Otra característica concomitante de la finitud es la polaridad, esto es, la escogencia entre positivo y negativo. Para que algo que se enuncia sea discutible tiene que ser o no 
ser, en el caso de una proposición; o bien, tiene que hacerse o no hacerse en el caso de una propuesta. En resumen, el elemento Finito combina la especificación de la polaridad con la referencia temporal o modal al evento de habla. En este sentido, constituye el componente verbal del Modo.

\section{Subsistemas del Sistema de Finito en bribri}

El elemento Finito en bribri circunscribe la cláusula de múltiples formas. En los siguientes apartados, se analizará cada uno de los subsistemas que conforman el Sistema de Finito. La distinción entre Sistema y subsistema depende de la perspectiva que se esté considerando. El Sistema de TIPO DE MODALIDAD, por ejemplo, es un sistema cuando se presente per se y, a la vez, un subsistema si se considera parte del Sistema de FINITO. Puesto que en los siguientes apartados se analizan los sistemas que conforman el Sistema de FINITO, estos se denominarán subsistemas, aunque pueden constituir sistemas per se.

\subsection{El subsistema de MODALIDAD del verbo en bribri}

Halliday (2004: 147) define los grados intermedios entre los polos positivo y negativo como MODALIDAD. Este sistema interpreta la región de incertidumbre que existe entre los dos extremos del Sistema de POLARIDAD: 'sí'/"no'. El espacio semántico codificado por la modalidad varía dependiendo de si la cláusula es una proposición o una propuesta.

En el caso de las proposiciones, existen dos tipos de posibilidades intermedias: (i) grados de probabilidad o posibilidad; (ii) grados de habitualidad. Estas dos escalas se definen en GSF como modalización.

Paralelamente, en el caso de las propuestas, existen dos tipos de posibilidades intermedias, dependiendo de la función de habla: (i) en el caso de una orden, existen grados de obligación; (ii) en una oferta existen grados de inclinación. Las escalas de obligación e inclinación se definen como modulación.

Las escalas de modalidad pueden expresarse en las lenguas de forma metafórica, o bien, congruente. Halliday (2004: 613) ejemplifica la realización metafórica de la escala de probabilidad mediante la cláusula I don't believe that pudding will ever be cooked. En este caso, la cláusula mental cognitiva I don't believe constituye una realización metafórica de probabilidad, esto es, la probabilidad se realiza por medio de una cláusula mental como si fuera una figura de sentir (sensing) desde la perspectiva experiencial. Siendo metafórica, la cláusula I don't believe, además de proyectar otra cláusula (that pudding will ever be cooked), también funciona como Adjunto de Modo en la estructura de Modo, al igual que probably.

Otros ejemplos de realizaciones congruentes y metafóricas de la modalidad, en inglés, son: (i) I wouldn't... if I was you: orden, realizada congruentemente por medio de don't...!; (ii) I've a good mind to...: oferta modalizada, realizada congruentemente en maybe I'll...; (iii) she'd better...: orden modulada, realizada congruentemente en she should...

En cuanto a la función de la metáfora interpersonal, Halliday argumenta que:

Interpersonal metaphor is thus the hinge between the ideational and interpersonal modes of constructing the self. In the ideational mode we construe ourselves as conscious Sensers, while in the interpersonal mode we enact ourselves as speakers interacting with addressees; the metaphor brings the two together in such a way that the ideational construal stands for the interpersonal enactment. (2006: 583) 
Las realizaciones congruentes de la modalidad se dan cuando esta es expresada por medio del elemento Finito o de un Adjunto de Modo. La realización es congruente porque se da en la estructura de Modo, es decir, en la metafunción interpersonal. En las realizaciones metafóricas de la modalidad, las escalas de modalización y modulación no se expresan mediante recursos pertenecientes a la estructura del Modo, sino que estas se realizan por medio de distintos tipos de cláusula, en la metafunción experiencial. Un ejemplo de realización metafórica y congruente de yo quiero dormir, en la escala de inclinación en bribri, es el que se muestra en la siguiente figura:
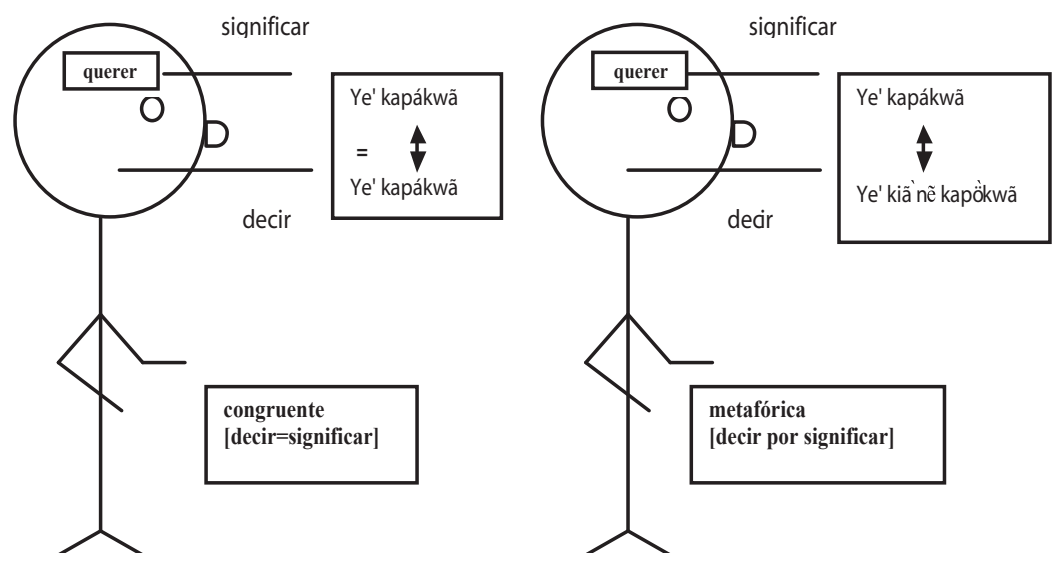

Figura 1. Realizaciones congruentes y metafóricas de la inclinación (basado en Matthiessen y Painter 1997: 69)

Como se puede observar en la figura 1, el bribri presenta dos distintas realizaciones en la escala de inclinación: una congruente, en la que la volición se expresa por medio del sufijo desiderativo -ák; y una metafórica, en la que la deseabilidad se realiza por medio de una cláusula mental desiderativa con el verbo kiã̀nẽ. En el caso de la realización congruente, el sufijo desiderativo es parte del elemento Finito y expresa modalidad, es decir, su análisis recae en la perspectiva de la cláusula como intercambio. Sin embargo, la realización metafórica se expresa en términos experienciales por medio de una cláusula mental.

El Sistema de TIPO DE MODALIDAD en bribri puede representarse de la siguiente forma:

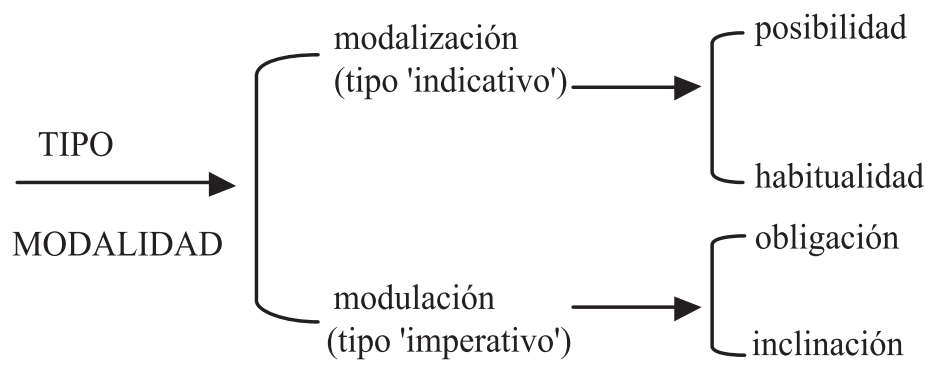

Sistema 1. TIPO DE MODALIDAD (Halliday 2004: 618)

En los siguientes apartados, se describen los recursos que, en los textos analizados, realizan de manera congruente o metafórica los tipos de modalidad que aparecen en el sistema 1. 


\subsubsection{Modulación: inclinación y obligatoriedad}

La tabla 1 muestra las distintas realizaciones de las escalas de inclinación y obligatoriedad encontradas en los textos objeto de análisis:

Tabla 1. Modulación

\begin{tabular}{|c|c|c|}
\hline & \multicolumn{2}{|r|}{ Modulación } \\
\hline & Inclinación & Obligatoriedad \\
\hline $\begin{array}{l}\text { Realización } \\
\text { congruente }\end{array}$ & $\begin{array}{l}\text { no, es que ye' kapákwã } \\
\text { no es que } 1 \mathrm{~S} \text { quiero-dormir } \\
\text { No, es que tengo sueño }(\mathrm{T} 2: 4)^{3}\end{array}$ & $\begin{array}{l}\text { e' kốwötã ulà klöwẽ wẽke ulítanẽ sếrãã } \\
\text { eso debe mano pone todos todo } \\
\text { Se debe poner las manos en todas las cosas (T4:5) }\end{array}$ \\
\hline \multirow[b]{2}{*}{$\begin{array}{l}\text { Realización } \\
\text { metafórica }\end{array}$} & \multicolumn{2}{|c|}{$\begin{array}{l}\text { kṍrke ie'pa ẽ̀n ã i katák, e'tã ie'pa i ttéwã katè } \\
\text { cuando 3PL hígado en eso quieren-comer entonces 3PL eso matan para comérselo } \\
\text { Cuando quieren comer eso, entonces lo matan para comérselo (T2:57) }\end{array}$} \\
\hline & $\begin{array}{l}\text { ye' kĩ i kiànẽ tö ye'rö Bribriwak } \\
1 \mathrm{~S} \text { sobre eso quiero que } 1 \mathrm{~S} \text { soy } \\
\text { gente-bribri } \\
\text { Yo quiero ser bribri (T2:41) }\end{array}$ & - \\
\hline
\end{tabular}

La expresión de la inclinación puede tener en bribri una realización tanto congruente (T2:4), como metafórica, mediante una cláusula mental desiderativa (T2:41). Sin embargo, existe también la posibilidad de combinación entre realización congruente y metafórica: en efecto, en T2:57, la inclinación hacia algo se expresa de manera metafórica con una cláusula mental, por medio de (pos) ̃̇n $\tilde{a}^{2}$ (en mi hígado) y congruente, por medio del sufijo desiderativo -ák que es parte del Finito.

En cuanto a la obligación, existe sólo la posibilidad de una realización congruente, mediante la forma invariable kốwöt $\tilde{a}^{3}$.

\subsubsection{Modalización: posibilidad y habitualidad}

La tabla 2 muestra las distintas realizaciones de las escalas de inclinación y obligatoriedad encontradas en los textos objeto de análisis:

Tabla 2. Modalización

\begin{tabular}{|l|l|l|}
\hline \multirow{2}{*}{} & \multicolumn{2}{|c|}{ Modalización } \\
\cline { 2 - 3 } & Posibilidad & Habitualidad \\
\hline $\begin{array}{l}\text { Realización } \\
\text { congruente }\end{array}$ & $\begin{array}{l}\text { se' kẽ ã i yõ̀rpa } \\
\text { 1PL NEG para eso era-posible } \\
\text { Nosotros no hubiéramos podido (eliminarlos) } \\
\text { (T3:51) }\end{array}$ & $\begin{array}{l}\text { e' balo' yawèke ie' tã } \\
\text { eso chicha hacían 3S con } \\
\text { Ellos solían hacer chicha con él (T2:19) }\end{array}$ \\
\hline & $\begin{array}{l}\text { se' lörmĩ } \\
\text { 1PL podemos-reproducirnos } \\
\text { Nosotros sí pudimos multiplicarnos } \\
\text { (T3:52) }\end{array}$ & $\begin{array}{l}\text { ès Sibökãnẽ̀ balö } \\
\text { así Sibö trabaja } \\
\text { Así trabaja Sibö(T3:54) }\end{array}$ \\
\hline $\begin{array}{l}\text { Realización } \\
\text { metafórica }\end{array}$ & - & - \\
\hline
\end{tabular}


En cuanto a la modalización, las escalas de probabilidad y habitualidad en bribri no contemplan realizaciones metafóricas, pues ambas se realizan por medio de sufijos que forman parte del elemento Finito. En T3:51, la probabilidad se expresa por medio de - $p a$ cuando la polaridad de la cláusula es negativa; y por medio del sufijo -mĩ (cláusula 52) cuando la cláusula es positiva. Este sufijo también tiene valor incoativo ${ }^{4}$ en el Sistema de FASE DEL TIEMPO. Jara Murillo (1995c: 10) indica que -mĩ, además de marcar el aspecto incoativo en el verbo, también expresa futuro y modo potencial conjuntamente. El imperfectivo de voz media también constituye un recurso de la lengua para expresar probabilidad ${ }^{5}$ o posibilidad de hacer algo en un determinado contexto. En los textos analizados, el imperfectivo de voz media es un recurso utilizado frecuentemente para expresar posibilidad o imposibilidad, como se puede observar en los siguientes ejemplos.

\begin{tabular}{|c|c|c|}
\hline $\begin{array}{l}\text { Kë̀ } \\
\text { NEG }\end{array}$ & \multicolumn{2}{|c|}{$\begin{array}{l}\text { kùrwã } \\
\text { podía-beberse }\end{array}$} \\
\hline Finito & \multicolumn{2}{|c|}{ Predicador^Finito } \\
\hline Modo & Residuo & Modo \\
\hline \multicolumn{3}{|c|}{ No se podía beber de él (T3:70) } \\
\hline
\end{tabular}

(2)

\begin{tabular}{|c|c|c|c|c|}
\hline $\begin{array}{l}\mathrm{se}^{\prime} \\
1 \mathrm{PL}\end{array}$ & $\begin{array}{l}\text { kề } \\
\text { NEG }\end{array}$ & $\begin{array}{l}\tilde{A} \\
\text { para }\end{array}$ & $\begin{array}{l}\mathrm{i} \\
\text { eso }\end{array}$ & $\begin{array}{l}\text { yörpa } \\
\text { era-posible }\end{array}$ \\
\hline Suj- & Finito & -eto & Complemento & Predicador $^{\wedge}$ Finito \\
\hline \multicolumn{3}{|c|}{ Modo } & Residuo & Modo \\
\hline
\end{tabular}

En (1), no hay ningún Sujeto o Agente explícito, puesto que el verbo aparece en voz media. En (2), sin embargo, existe un Participante involucrado marcado como dativo mediante la posposición ã (Jara Murillo y García Segura 2009). Desde la perspectiva interpersonal, dicho Participante es el Sujeto de la cláusula, es decir, el elemento que está posibilitado o imposibilitado para hacer algo. Desde la perspectiva experiencial, este Participante se define como Posibilitador (cf. Cruz Volio, inédito: 52).

Por lo que atañe a la escala de habitualidad, esta escala en bribri se realiza por medio de dos formas verbales definidas como imperfecto primero e imperfecto segundo (Constenla Umaña et al. 1998: 82, 90); o imperfectivo simple e imperfectivo habitual respectivamente (Jara Murillo y García Segura, en prensa). Constenla Umaña et al. (1998: 82) indican que el imperfecto primero, es decir, la forma kãnèbalö en T3:54 (tabla 2), se puede emplear para referirse a acontecimientos de aspecto imperfectivo independientemente del tiempo, aunque se prefiere su uso en las cláusulas que especifican la forma en la que algo se lleva a cabo habitualmente. El imperfecto segundo (Constenla Umaña et al. 1998: 90) se forma añadiendo el sufijo -ke a la forma de imperfecto primero como en yawèke en T1:19 (tabla 2), y, de acuerdo con los autores, expresa la condición habitual de acciones o estados, tanto referido al presente, como al pasado y al futuro. Al respecto, Jara Murillo afirma que: "La mayoría de las situaciones presente y futuras en el bribri de Coroma se expresan mediante la forma habitual en lugar de la forma imperfectiva simple" (1995c: 9).

La siguiente figura representa una aproximación de cómo se construye, en bribri, el espacio semántico que existe entre los polos ‘sí’/‘no', a partir de los datos extraídos del análisis de los textos: 
MODALIZACIÓN

[posibilidad]

$-\mathrm{mi}$

sufijo de
posibilidad

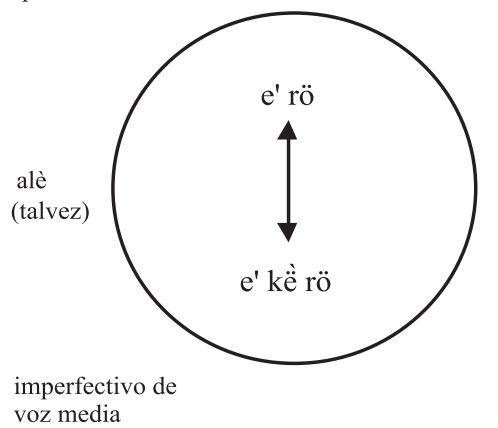

[habitualidad]
MODULACIÓN

[obligación] [inclinación]

positivo

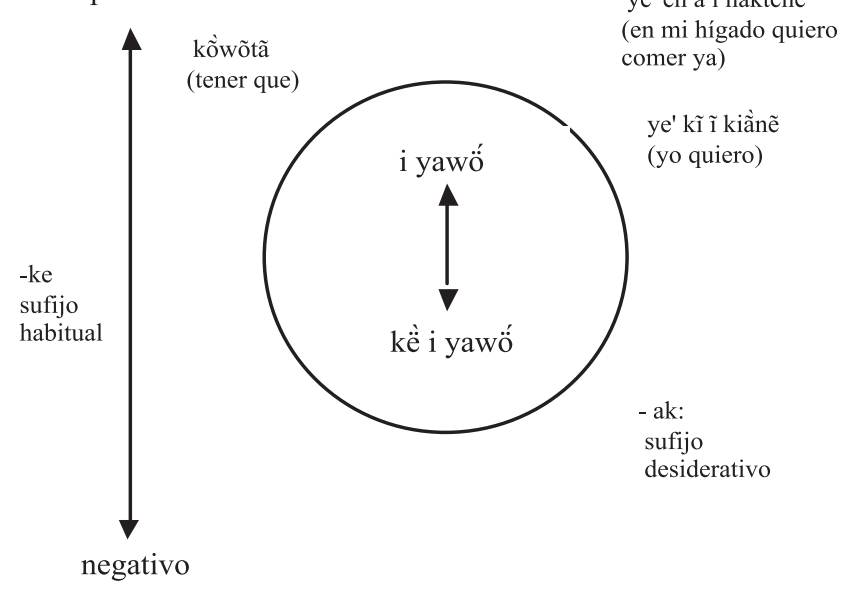

Figura 2. Relación entre polaridad, modalidad y MODO (con base en Halliday 2004: 619)

\subsection{El subsistema de EXPECTATIVA DEL HABLANTE en relación con la temporalidad}

El Finito puede realizar simultáneamente por lo menos dos opciones del Sistema de FINITO en bribri:

(3)

\begin{tabular}{|c|c|c|}
\hline $\begin{array}{l}\text { ye' ẽn ã } \\
1 S \text { hígado en }\end{array}$ & $\begin{array}{l}\mathrm{i} \\
\text { eso }\end{array}$ & $\begin{array}{l}\text { ñắktche } \\
\text { quiero-comer-ya }\end{array}$ \\
\hline Sujeto & Complemento & Predicador^Finito \\
\hline Modo & Residuo & Modo \\
\hline
\end{tabular}

En T3:17, en el verbo ñã́ ktche, el Finito expresa: (i) modalidad y (ii) temporalidad relativa a la expectativa del hablante. Más específicamente, el sufijo desiderativo -ák expresa la inclinación (modalización) del hablante al querer comer y -tche indica la expectativa positiva del hablante en cuanto a la temporalidad en la que se va a realizar el evento. Según Constenla Umaña et al. (1998: 85), el sufijo -tche se une a los verbos a la derecha de otros sufijos para expresar la idea que denota en castellano el adverbio ya. Los autores lo definen como sufijo anticipativo y especifican que se puede emplear exclusivamente en cláusulas afirmativas.

Desde la perspectiva sistémico-funcional, -tche se considera como una expresión de la expectativa del hablante con respecto al tiempo del evento del que se está hablando (Halliday 2004: 128). La expectativa del hablante puede expresarse en bribri conjuntamente con el tiempo primario, además de la modalidad, como en el ejemplo (3). Otras instancias de la expresión de la expectativa son los siguientes: 
(4)

\begin{tabular}{|c|c|c|c|}
\hline $\begin{array}{l}\text { íñẽ tsốlĩ } \\
\text { hoy tarde }\end{array}$ & \begin{tabular}{|l}
$\mathrm{se}$ \\
1PL \\
\end{tabular} & $\begin{array}{l}\text { i } \\
\text { eso }\end{array}$ & $\begin{array}{l}\text { yèkètche } \\
\text { ya-vamos-a-beber }\end{array}$ \\
\hline Adjunto & Sujeto & Complemento & Predicador ${ }^{\wedge}$ Finito \\
\hline Residuo & Modo & Residuo & Modo \\
\hline
\end{tabular}

(5)

\begin{tabular}{|l|l|}
\hline $\begin{array}{l}\text { kồre ñẽ' } \\
\text { todo aquello }\end{array}$ & $\begin{array}{l}\text { rpánẽtche } \\
\text { ya-amontonaron }\end{array}$ \\
\hline Complemento & \multicolumn{2}{|l|}{ Predicador^Finito } \\
\hline Residuo & Modo \\
\hline \multicolumn{2}{|l|}{ Todo aquello ya está amontonado (T4:14) } \\
\hline
\end{tabular}

En yèkètche y rpánẽtche el Finito expresa futuro cercano y pasado respectivamente, además de la expectativa del hablante. La contraparte negativa en la temporalidad relativa a la expectativa del hablante es el sufijo -iã. Constenla Umaña et al. (1998: 85) definen -iã como sufijo de pertinencia actual que puede expresar la idea de 'ya no' o 'todavía'. En los textos analizados se han encontrado ejemplos de este sufijo unido al Predicador con el significado de 'todavía' (véase ejemplo 6) y también bajo la forma de Adjunto de Modo con el significado de ‘ya no' en cláusulas negativas (7 y 8) y como Adjunto de Modo en cláusulas afirmativas con el significado de 'todavía' (9):

(6)

\begin{tabular}{|c|c|c|c|}
\hline $\begin{array}{l}\text { mî̀ kã } \\
\text { cuando }\end{array}$ & $\begin{array}{l}\mathrm{se}^{\prime} \\
1 \mathrm{PL}\end{array}$ & \multicolumn{2}{|c|}{$\begin{array}{l}\text { bitsồkeiã } \\
\text { todavía-ayuna }\end{array}$} \\
\hline & Sujeto & \multicolumn{2}{|c|}{ Predicador^Finito } \\
\hline & Modo & Residuo & Modo \\
\hline \multicolumn{4}{|c|}{ Cuando uno está en pleno ayuno (todavía ayuna) (T5:4) } \\
\hline
\end{tabular}

(7)

\begin{tabular}{|c|c|c|c|c|c|}
\hline $\begin{array}{l}\text { be' } \\
2 S\end{array}$ & \begin{tabular}{|l} 
lè \\
tal vez
\end{tabular} & $\begin{array}{l}\text { ye' } \\
1 S\end{array}$ & $\begin{array}{l}\text { lè } \\
\text { tal vez }\end{array}$ & $\begin{array}{l}\text { kë kũ } \\
\text { NEG estemos }\end{array}$ & $\begin{array}{l}\text { iã } \\
\text { más }\end{array}$ \\
\hline Sujeto & $\begin{array}{l}\text { Adjunto } \\
\text { modo }\end{array}$ & Sujeto & $\begin{array}{l}\text { Adjunto } \\
\text { modo }\end{array}$ & Finito $\wedge$ Predicador & $\begin{array}{l}\text { Adjunto } \\
\text { modo }\end{array}$ \\
\hline \multicolumn{4}{|c|}{ Modo } & Residuo & Modo \\
\hline
\end{tabular}

(8)

\begin{tabular}{|c|c|c|c|c|}
\hline $\begin{array}{l}\text { kë̀ } \\
\text { NEG }\end{array}$ & $\begin{array}{l}\text { wồyök } \\
\text { mal-agüero }\end{array}$ & $\begin{array}{l}\text { sũwẽ̀ kũ } \\
\text { no-vemos }\end{array}$ & $\begin{array}{l}\text { iã } \\
\text { más }\end{array}$ & $\begin{array}{l}\text { sö } \\
\text { 1PL.ERG }\end{array}$ \\
\hline Finito & Complemento & Predicador $^{\wedge}$ Finito & $\begin{array}{l}\text { Adjunto } \\
\text { Modo }\end{array}$ & Sujeto \\
\hline Modo & \multicolumn{2}{|l|}{ Residuo } & \multicolumn{2}{|c|}{ Modo } \\
\hline
\end{tabular}

(9)

\begin{tabular}{|l|l|l|l|}
\hline $\begin{array}{l}\text { se' } \\
1 \mathrm{PL}\end{array}$ & $\begin{array}{l}\text { yörke } \\
\text { preparan }\end{array}$ & $\begin{array}{l}\text { èrpa } \\
\text { apenas }\end{array}$ & $\begin{array}{l}\text { e' iã } \\
\text { eso todavía }\end{array}$ \\
\hline Sujeto & Predicador^Finito & Adjunto Modo & Adjunto Modo \\
\hline Modo & Residuo & Modo \\
\hline
\end{tabular}


El subsistema de TEMPORALIDAD RELATIVA A LA EXPECTATIVA DEL HABLANTE puede delinearse de la siguiente manera:

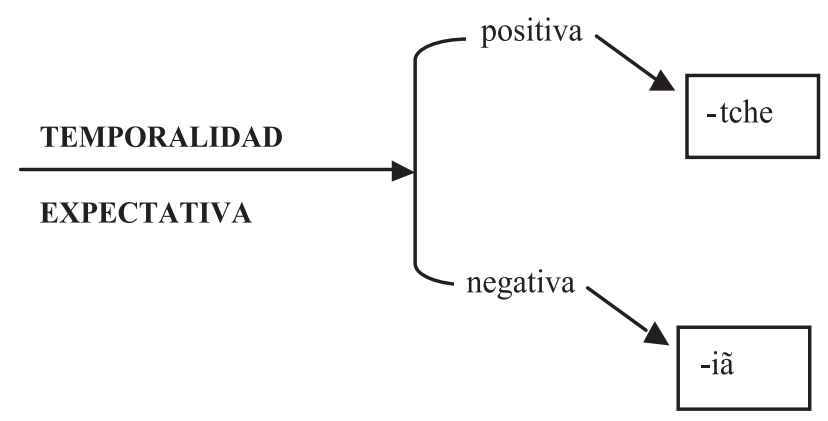

\section{Sistema 2. TEMPORALIDAD RELATIVA A LA EXPECTATIVA}

DEL HABLANTE

\subsection{El subsistema de FASE}

En cuanto al subsistema de FASE, realizado en el elemento Finito, este presenta exclusivamente una entrada: (i) fase del tiempo. La fase del tiempo cuenta con tres opciones de escogencia: incoativo, durativo y conclusivo; que representan las tres posibles opciones de escogencia del subsistema:

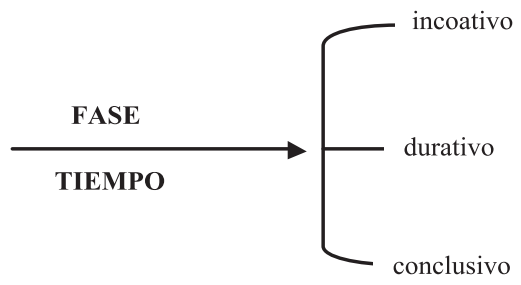

Sistema 3. FASE DEL TIEMPO

\subsubsection{FASE DEL TIEMPO: incoativo}

Tabla 3. Fase del tiempo incoativo

\begin{tabular}{|l|l|}
\hline \multicolumn{2}{|c|}{ Fase del tiempo } \\
\hline \multirow{2}{*}{ Incoativo } & -mĩ: \\
& ie' chòwàmĩ \\
& 3S se-empezó-a-perder \\
& Se empezó a perder (T2:73) \\
\cline { 2 - 3 } & ẽ' tchöwã + infinitivo: \\
& E'tã ie' ẽ' tchöwã ñẽ' kố yawök \\
& entonces 3S REFL puso aquel mundo hacer \\
& Así pues, él se puso a hacer el mundo (T3:55) \\
\cline { 2 - 3 } & imperfectivo simple + ẽ' tchèwã: \\
& ie' r í́kyök yawè tchèwã \\
& 3S ERG tierra hacer se-puso \\
& Él se puso a hacer la tierra (T3:58) \\
\hline
\end{tabular}


Jara Murillo (1995c), al describir los recursos del bribri para expresar el aspecto incoativo o ingresivo, indica entre ellos el sufijo - $m \tilde{\imath}$ y la construcción con el verbo de significación ingresiva $\tilde{e}^{\prime} t c h o ̈ k w \tilde{a}$ y el verbo principal en forma imperfectiva. Como se muestra en la tabla 3, en los textos se han encontrado los dos recursos mencionados por la autora. Cabe destacar que el verbo de significación ingresiva $\tilde{e}^{\prime}$ tchökwã puede estar seguido también de un infinitivo e incluso se ha encontrado en uno de los textos un caso en el que la forma imperfectiva simple precede este verbo. Es interesante observar, además, que la semántica del verbo bribri puede añadir significados adicionales a la fase de tiempo incoativa:

\begin{tabular}{|c|c|c|c|c|}
\hline $\begin{array}{l}\text { pë' } \\
\text { persona }\end{array}$ & \multicolumn{2}{|c|}{$\begin{array}{l}\text { burùtchö } \\
\text { se-apresuraba }\end{array}$} & $\begin{array}{l}\text { Krò } \\
\text { gallo }\end{array}$ & $\begin{array}{l}\text { Lök } \\
\text { cocinar }\end{array}$ \\
\hline Sujeto & \multicolumn{2}{|c|}{ Predicador^Finito } & Complemento & Predicador \\
\hline Modo & Residuo & Modo & Residuo & Modo \\
\hline \multicolumn{5}{|c|}{ Abèbulu se apresuraba a cocinar el gallo (T2:19) } \\
\hline
\end{tabular}

En (10), el Predicador burùtchö expresa el sentido de empezar a hacer algo de manera apresurada, en este caso 'cocinar' (lök). Un complejo verbal como burùtchö lök puede analizarse desde la perspectiva sistémico-funcional, en términos de elaboración, extensión e incremento. Halliday (2004: 497) sostiene que un complejo verbal está formado por un grupo primario (i.e. burùtchö), que conlleva el Modo de la cláusula (Predicador^Finito); y un grupo secundario, que realiza el tipo de proceso de la cláusula.

El grupo primario y el secundario pueden estar relacionados por medio de varios tipos de relaciones hipotácticas, que corresponden bastante sistemáticamente a los diferentes patrones del complejo clausular. En efecto, el subsistema de FASE, que en el presente análisis es parte del Sistema de FINITO en bribri, se considera, según Halliday, como la elaboración de un proceso: las entradas de incoativo, durativo, conclusivo y progresivo, deben entenderse desde esta perspectiva como la elaboración de un proceso de 'devenir'.

Entre las relaciones hipotácticas que pueden caracterizar el complejo verbal, cabe tomar en cuenta, en el caso del bribri, lo que Halliday define como el incremento de un proceso:

\footnotetext{
The basic notion is that of 'be (circumstantial) + do', for example help to do 'do being-with (someone)'. [...] Here the primary verbal group is again not a separate process; but this time it is a circumstantial element in the process expressed by the secondary verbal group. If Alice ventured to ask something, this means she did ask it; but she did so tentatively. (Halliday 2004: 503)
}

El incremento de un proceso en un grupo verbal se define como MODULACIÓN. Este subsistema representaría en bribri una de las entradas del Sistema de COMPLEJO VERBAL. Sin embargo, puesto que la presente investigación se limita a considerar aspectos propios de la cláusula simple, este subsistema no se incluirá en el Sistema de FINITO; contrariamente al subsistema de FASE, que se manifiesta en bribri en la estructura de Modo de la cláusula simple Predicador^Finito. Aunque el subsistema de MODULACIÓN no se tomará en cuenta, parece relevante proporcionar una categorización de complejos verbales restringida a los ejemplos obtenidos a partir de los textos, utilizando algunas de las categorías propuestas por Halliday (2004: 504): 
Tabla 4. Instancias de incremento del proceso en el complejo verbal

\begin{tabular}{|c|c|}
\hline \multicolumn{2}{|r|}{ Incremento del proceso } \\
\hline Categorías & Ejemplos \\
\hline \multirow[t]{3}{*}{ Manera } & $\begin{array}{l}\text { pë' burùtchö krò lök } \\
\text { persona se-apresuraba gallo cocinar } \\
\text { Abèbulu se apresuraba a cocinar el gallo } \\
\text { (T3:19) }\end{array}$ \\
\hline & $\begin{array}{l}\text { e'tã iök ie' römĩ tur èẽ? } \\
\text { entonces para-qué 3S pasaba-por corría ahí } \\
\text { ¿Entonces por qué andaba corriendo por ahí? } \\
\text { (T2:27) }\end{array}$ \\
\hline & $\begin{array}{l}\text { se' kapö̀e tër wî́ẽ tër sík kĩ } \\
\text { 1PL duerme está-echado allá echado hoja sobre } \\
\text { Uno duerme sobre hojas } \\
\text { (T5:8) }\end{array}$ \\
\hline \multirow[t]{4}{*}{ Finalidad } & $\begin{array}{l}\text { e'tã ie'pa i ttéwã katè } \\
\text { entonces 3PL eso mataron comen } \\
\text { Entonces lo matan para comérselo } \\
\text { (T3:57) }\end{array}$ \\
\hline & $\begin{array}{l}\text { e'tã ye' kiố blàtök } \\
\text { entonces 1S llame repartir } \\
\text { "Ah bueno, llámeme para repartirlo" } \\
\text { (T3:21) }\end{array}$ \\
\hline & $\begin{array}{l}\text { e' rö sí i kále, s ttó mõwẽ̀ cha. } \\
\text { eso es sí eso 1PL hablamos imitamos pues } \\
\text { Eso sí que es una broma, tratar de imitarnos } \\
\text { (T2:85) }\end{array}$ \\
\hline & $\begin{array}{l}\text { se' ã e' bulùkala sề ò̀wã i di } \\
\text { 1PL para eso traicionó enfrió } 3 \mathrm{~S} \text { ERG } \\
\text { Sibö lo enfrió para nosotros } \\
\text { (T3:70) }\end{array}$ \\
\hline \multirow[t]{4}{*}{$\begin{array}{l}\text { Finalidad: } \\
\text { (con verbos de movimiento) }\end{array}$} & $\begin{array}{l}\text { be' i chè tö barátsèke i ulàtök } \\
\text { persona eso dice } 2 \text { S eso dice que venía eso repartir } \\
\text { "Usted dijo que lo quería repartir" } \\
\text { (T3:26) }\end{array}$ \\
\hline & $\begin{array}{l}\text { ie' ẽ duèsẽ bikèitsök } \\
\text { 3S REFL paró pensar } \\
\text { entonces paró a pensar } \\
\text { (T2:75) }\end{array}$ \\
\hline & $\begin{array}{l}\text { i tsố ù, ie' kố yawè dèe } \\
3 \mathrm{~S} \text { primera casa } 3 \mathrm{~S} \text { mundo hace fue } \\
\text { El hizo el mundo como su primera casa } \\
\text { (T3:65) }\end{array}$ \\
\hline & $\begin{array}{l}\text { èẽ î́kyök wák pë' láköl tãì̀ e' tsũk ie' dềdë } \\
\text { allá tierra dueña mujer grande esa traer } 3 \mathrm{~S} \text { fue } \\
\text { Fue allá adonde la madre a traer a la niña tierra. } \\
\text { (T3:59) }\end{array}$ \\
\hline
\end{tabular}




\begin{tabular}{|l|l|}
\hline & $\begin{array}{l}\text { e' dàtsẽ se' ulà klöũ tsurù kĩ } \\
\text { esa llega 1PL mano poner cacao sobre } \\
\text { Llega a ponerle a uno la mano en el cacao } \\
\text { (T4:3) }\end{array}$ \\
\hline Finalidad: & krò dèmĩ tchër \\
(con estativos de posición) & gallo fue a-caer \\
& El gallo fue a caer por allá \\
& (T3:33) \\
\cline { 2 - 2 } & Mì̃ãttchër \\
& fue-a-sentarse \\
& Siböfue a sentarse \\
& (T3:29) \\
\hline
\end{tabular}

El ejemplo T3:57, en la tabla 4, representa una instancia de lo que Constenla Umaña et al. (1998: 83) definen como el uso aislado del imperfectivo como complemento de finalidad. Se puede observar que los ejemplos de incremento del proceso extraídos de los textos se forman por medio de la yuxtaposición de dos formas verbales, ya sea ambas flexionadas (T3:27, T3:57, T3:70, T2:85) o una flexionada y la otra en infinitivo (T3:19, T3:21, T3:26, T2:75). Jara Murillo (1995c: 15) las define como infinitivos en función de finalidad.

\subsubsection{FASE DEL TIEMPO: durativo}

Tabla 5. Fase del tiempo durativo

\begin{tabular}{|c|c|}
\hline & Fase del tiempo \\
\hline \multirow[t]{7}{*}{ Durativo } & $\begin{array}{l}\text { existencial perfectivo (bák) + infinitivo: } \\
\text { Ìke ès, Sibö̀ bák kố i' kĩ ñì̀ppök Sòrbulu tã } \\
\text { pues así Sibö estuvo mundo este sobre pelear Sòrbulu con } \\
\text { Así pues, Sibö estuvo en este mundo peleando con Sòrbulu (T3:1) }\end{array}$ \\
\hline & $\begin{array}{l}\text { infinitivo + estativo de posición (tchër): } \\
\text { E' kõs e' wàbalök ie' tchër } \\
\text { eso todo eso hacer } 3 \mathrm{~S} \text { estaba } \\
\text { Todo esto estuvo haciendo él (T3:48) }\end{array}$ \\
\hline & $\begin{array}{l}\text { estativo de posición (tër) + infinitivo: } \\
\text { Wẽ́ ie' tër e' wàbalök bë këbëla ie? } \\
\text { adónde } 3 \mathrm{~S} \text { estaba-echado eso hacer diablo muchacho.DIM como } \\
\text { ¿Adónde estaba haciendo esas cosas ese pequeño diablo? (T2:66) }\end{array}$ \\
\hline & $\begin{array}{l}\text { tso' + infinitivo: } \\
\text { e'tã sa' tso' kốpàkök. } \\
\text { entonces } 1 \mathrm{PL} \text { estamos conversar } \\
\text { Entonces nos pusimos a conversar (T2:36) }\end{array}$ \\
\hline & $\begin{array}{l}\text { estativo de posición (tẽr) + infinitivo: } \\
\text { E'tã i tẽ́rkerak nãmã kalíuuk. } \\
\text { entonces } 3 \text { PL estaban pez enganchando } \\
\text { Solían ir a pescar (T2:59) }\end{array}$ \\
\hline & $\begin{array}{l}\text {-ke: } \\
\text { ie' kốchöke pe' tã } \\
\text { 3S mentía persona con } \\
\text { Él le estaba mintiendo }(\mathrm{T} 3: 30)\end{array}$ \\
\hline & $\begin{array}{l}\text { imperfectivo simple (imperfecto primero): } \\
\text { Ie' kốchö ie' èköl shtèwã tottóla. } \\
\text { 3S mentía 3S otro engañaba fácil.DIM } \\
\text { Sibö mentía, solamente estaba engañando al otro (T3:18) }\end{array}$ \\
\hline
\end{tabular}


Acerca del aspecto durativo, Jara Murillo (1995c: 21) indica que el sufijo -ke, aparte de ser un marcador de habitual también codifica situaciones durativas en presente o pasado, sean habituales o no. Entre los recursos descritos por la autora, también aparecen la construcción tso' + infinitivo y la forma verbal imperfectiva + estativo de posición. En la tabla 5, aparece una instancia (T3:30) del sufijo -ke con valor durativo, además de la presencia en los textos de los siguientes recursos: la construcción estativo de posición + infinitivo (T2:66), cuyo orden también aparece invertido (T3:48), el uso del imperfectivo simple (T3:18) y la forma perfectiva (bák) del existencial $t s o^{\prime}$ seguido por el verbo en infinitivo.

\subsubsection{FASE DEL TIEMPO: conclusivo}

Tabla 6. Fase del tiempo conclusivo

\begin{tabular}{|c|c|}
\hline & Fase del tiempo \\
\hline \multirow[t]{2}{*}{ Conclusivo } & $\begin{array}{l}\text { wồñõr: } \\
\text { e' wönñor tás, } \\
\text { eso dejó-de-hacer IDEOF } \\
\text { Sibö dejó de hacer malos agüeros (T3:38) }\end{array}$ \\
\hline & $\begin{array}{l}\text { imperfectivo simple + ồnẽ: } \\
\text { e' mĩ̀ kã be' tsuru' kkuè ồnẽ, bàwềke } \\
\text { eso cuando } 2 \mathrm{~S} \text { cacao tuesta terminó calienta } \\
\text { Cuando usted terminó de tostar el cacao, (lo) calienta (T4:11) }\end{array}$ \\
\hline
\end{tabular}

Por lo que se refiere a la fase del tiempo conclusivo, solo se han encontrado dos instancias. En T3:38, el verbo wöñor tiene, en el contexto narrativo en el que se encuentra, el significado de 'dejar de hacer algo'. Sin embargo, este valor se le atribuye exclusivamente mediante el contexto, puesto que este verbo en bribri también significa 'iluminar'. Por otro lado, la construcción imperfectivo simple + õ̀nuk (caer) es un recurso de la lengua para expresar la opción 'conclusivo' en el subsistema FASE DEL TIEMPO.

\subsection{El subsistema de TIEMPO PRIMARIO}

El subsistema de TIEMPO PRIMARIO se refiere al tiempo al que el discurso hace referencia y constituye, por lo tanto, un tiempo contextualizado a partir de los textos. Este subsistema cuenta con tres posibles opciones de escogencia:

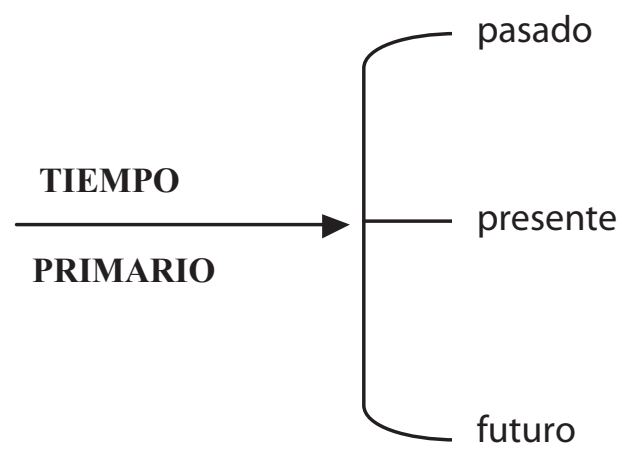

Sistema 4. TIEMPO PRIMARIO 
Por lo que se refiere al presente, en los textos se han encontrado dos recursos para la codificación de este tiempo primario: (i) la forma imperfectiva simple y (ii) la forma imperfectiva habitual (Jara Murillo 1995c: 23):

(11)

\begin{tabular}{|l|l|l|l|}
\hline $\begin{array}{l}\mathrm{e}^{\prime} \\
\text { esa }\end{array}$ & $\begin{array}{l}\text { dàtsẽ } \\
\text { viene }\end{array}$ & $\begin{array}{l}\text { se' ulà } \\
\text { 1PL mano }\end{array}$ & $\begin{array}{l}\text { Klöũk } \\
\text { Poner }\end{array}$ \\
\hline Sujeto & Predicador^${ }^{\wedge}$ Finito & Complemento & Predicador \\
\hline Modo & Residuo $\quad$ Modo & Residuo \\
\hline \multicolumn{4}{l|}{ esa viene a hacerle a uno la ceremonia de ponerle la mano $(\mathrm{T} 4: 1)$} \\
\hline
\end{tabular}

(12)

\begin{tabular}{|c|c|c|c|c|}
\hline $\begin{array}{l}\text { Kàlwö } \\
\text { semillas }\end{array}$ & $\begin{array}{l}\text { ë } \\
\text { sólo }\end{array}$ & \multicolumn{2}{|l|}{$\begin{array}{l}\text { Katèke } \\
\text { Comen }\end{array}$} & $\begin{array}{l}\text { Wî́kkëbulu } \\
\text { ese-tamaño.INT }\end{array}$ \\
\hline Complemento & Adjunto Modo & \multicolumn{2}{|c|}{ Predicador^Finito } & Adjunto \\
\hline Residuo & Modo & Residuo & Modo & Residuo \\
\hline
\end{tabular}

Cabe destacar, como se ha mencionado anteriormente, que en la mayoría de los casos el Finito en bribri codifica por lo menos dos opciones del Sistema de FINITO simultáneamente. En (11) y (12) el Finito expresa presente por medio de la forma imperfectiva simple e imperfectiva habitual respectivamente, además de expresar habitualidad, en la escala de modalización, en el subsistema de TIPO DE MODALIDAD.

En cuanto a la codificación de situaciones futuras, en los textos analizados se han encontrado diversos recursos: (i) la forma imperfectiva habitual -ke, (ii) la construcción con el verbo $m \tilde{\imath} k+$ infinitivo y (iii) el sufijo de futuro -ra:

\begin{tabular}{|l|l|l|l|}
\hline $\begin{array}{l}\text { íñ̃̃ tsốlĩ } \\
\text { hoy tarde }\end{array}$ & $\begin{array}{l}\text { se' } \\
\text { 1PL }\end{array}$ & $\begin{array}{l}\text { i } \\
\text { lo }\end{array}$ & $\begin{array}{l}\text { Yèkètche } \\
\text { ya-vamos-a-beber }\end{array}$ \\
\hline Adjunto & Sujeto & Complemento & Predicador^Finito \\
\hline Residuo & Modo & Residuo & Modo \\
\hline \multicolumn{4}{|l}{ Hoy en la noche empezaremos a beber $(\mathrm{T} 4: 14)$} \\
\hline
\end{tabular}

En (13), el Finito realiza, como ya se ha dicho, las opciones de dos subsistemas: (i) la opción futuro en el subsistema de TIEMPO PRIMARIO y (ii) la expectativa positiva del hablante en el subsistema TIEMPO RELATIVO A LA EXPECTATIVA DEL HABLANTE.

(14)

\begin{tabular}{|l|l|l|l|}
\hline $\begin{array}{l}\text { ye' } \\
1 \mathrm{~S}\end{array}$ & mĩ̀ & i & $\begin{array}{l}\text { lök } \\
\text { cocinar }\end{array}$ \\
\hline Sujeto & Finito & Complemento & Predicador \\
\hline Modo & Residuo \\
\hline Lo voy a cocinar $(\mathrm{T} 3: 10)$ & \\
\hline
\end{tabular}

La construcción con el verbo mìk + infinitivo, en (14), es otro recurso para la codificación de eventos futuros. La construcción es definida como una forma de futuro en Jara Murillo y García Segura (en prensa). 
(15)

\begin{tabular}{|l|l|l|}
\hline $\begin{array}{l}\text { be' } \\
2 S\end{array}$ & i & $\begin{array}{l}\text { sũẽràa } \\
\text { verá }\end{array}$ \\
\hline Sujeto & Complemento & Predicador^Finito \\
\hline Modo & Residuo & Modo \\
\hline \multicolumn{2}{|l}{ Usted verá (T2:72) } \\
\hline
\end{tabular}

Constenla Umaña et al. definen la forma de futuro en el ejemplo (13) como imperfecto futuro de certidumbre. Al respecto los autores indican que:

\footnotetext{
Para referirse al futuro cuando se quiere hacer hincapié en la seguridad que se tiene de lo que se predica va a ocurrir, se emplea el futuro de certidumbre, forma que, cuando es afirmativa, se constituye añadiendo al imperfecto primero el sufijo -dâ (variante empleada cuando precede r o n) -râ (variante usada en los demás casos). (1998: 84)
}

Los autores sostienen que una traducción al español de la forma de futuro de certidumbre sería, en el caso de la forma que aparece en (13), 'usted ha de ver'.

Jara Murillo (1995c: 23) indica como recursos para expresar situaciones futuras la gramaticalización del verbo dök, la forma imperfectiva habitual y el sufijo -mĩ, que expresa indistintamente acción futura y modo potencial. Constenla Umaña et al. definen esta forma como imperfecto potencial y afirman que se utiliza para expresar la idea de contingencia, tanto en el pasado como en el futuro (1998: 111).

En cuanto a la opción 'pasado', en el subsistema de TIEMPO PRIMARIO, se han encontrado cinco recursos básicos: (i) el perfectivo de voz media, (ii) el imperfectivo de voz media, (iii) el imperfectivo simple, (iv) el perfectivo y (v) la forma anterior:

\begin{tabular}{|l|l|l|}
\hline $\begin{array}{l}\text { Wö́ki } \\
\text { Cabeza }\end{array}$ & $\begin{array}{l}\text { yawènẽ } \\
\text { hizo }\end{array}$ & $\begin{array}{l}\text { nãmû̀ sũ̀ë } \\
\text { tigre parecido.INT }\end{array}$ \\
\hline Adjunto & Predicador^Finito & Complemento \\
\hline Residuo & Modo & Residuo \\
\hline \multicolumn{2}{|l}{ de su cabeza hizo algo parecido a un tigre $(\mathrm{T} 3: 40)$} \\
\hline
\end{tabular}

\begin{tabular}{|l|l|l|}
\hline $\begin{array}{l}\text { Chakà } \\
\text { Carne }\end{array}$ & $\begin{array}{l}\text { yawèlur } \\
\text { hizo }\end{array}$ & $\begin{array}{l}\text { òrke bukuë' ò̀rke } \\
\text { grita sapo grita }\end{array}$ \\
\hline Adjunto & Predicador^Finito & Complemento \\
\hline Residuo & Modo & Residuo \\
\hline Lo mató y de su carne hizo ese sapo que grita (T3:42) \\
\hline
\end{tabular}

Los ejemplos (16) y (17) muestran la forma perfectiva e imperfectiva respectivamente del verbo yawök (hacer) ${ }^{6}$.

\begin{tabular}{|l|l|l|l|}
\hline $\begin{array}{l}\text { ie' } \\
\text { 3S }\end{array}$ & $\begin{array}{l}\text { Sòrbulu e' } \\
\text { Sòrbulu ese }\end{array}$ & $\begin{array}{l}\text { ẽ̀ồwã } \\
\text { eliminó }\end{array}$ & $\begin{array}{l}\text { se' yökĩ } \\
\text { 1PL antes }\end{array}$ \\
\hline Sujeto & Complemento & Predicador ${ }^{\wedge}$ Finito & Adjunto \\
\hline Modo & Residuo & Modo & Residuo \\
\hline \multicolumn{2}{|l|}{ Sibö eliminó a Sòrbulu antes de aparecer nosotros (3:44) } \\
\hline
\end{tabular}


(19)

\begin{tabular}{|l|l|l|}
\hline $\begin{array}{l}\text { Nãũ̀chakepa } \\
\text { Nãù̀chakepa }\end{array}$ & $\begin{array}{l}\text { ẽ̀wẽ̀wã } \\
\text { eliminó }\end{array}$ & $\begin{array}{l}\text { i di } \\
\text { 3S ERG }\end{array}$ \\
\hline Complemento & Predicador^Finito & Sujeto \\
\hline Residuo & Modo \\
\hline \multicolumn{2}{|l}{ Eliminó a los Nãũ̀chakepa (3:45) } \\
\hline
\end{tabular}

Paralelamente, los ejemplos (18) y (19) muestran la forma perfectiva remota e imperfectiva simple, respectivamente, del verbo ẽ'õ'wã (eliminar) ${ }^{7}$.

(20)

\begin{tabular}{|c|c|c|c|}
\hline $\begin{array}{l}\text { Kë̀ } \\
\text { NEG }\end{array}$ & \multicolumn{2}{|l|}{$\begin{array}{l}\text { sũule } \\
\text { ha-visto }\end{array}$} & $\begin{array}{l}\text { ie' } \\
3 \mathrm{~S}\end{array}$ \\
\hline Finito & \multicolumn{2}{|c|}{ Predicador^Finito } & Sujeto \\
\hline Modo & Residuo & Mod & \\
\hline \multicolumn{4}{|c|}{ No los ha visto } \\
\hline
\end{tabular}

En el ejemplo (20), la forma verbal sũule constituye un ejemplo de lo que Constenla Umaña et al. (1998: 91) definen como forma anterior. Los autores argumentan que el sufijo -ule es un indicador de anterioridad y que las formas anteriores son básicamente intransitivas, puesto que se construyen a partir del tema de voz media. Además, como se puede observar en el mismo ejemplo, en bribri el concepto de 'conocer' se expresa mediante la forma anterior del verbo 'ver' (sãük $\rightarrow$ sũule). La cláusula debe entenderse, por lo tanto, como 'no los conoce (a los bribris)'.

\subsection{El subsistema de POLARIDAD}

El subsistema de POLARIDAD cuenta con dos posibles opciones de escogencia: positivo y negativo. En el espacio semántico que existe entre los dos polos se desarrolla el subsistema de MODALIDAD:

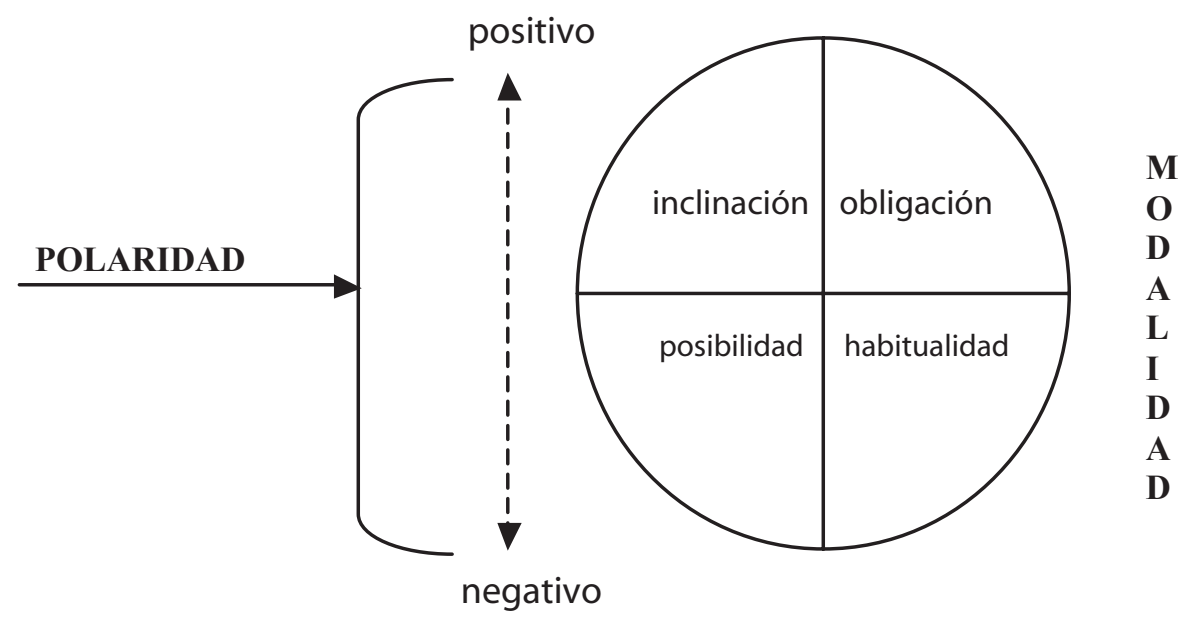

En la lengua bribri, la polaridad negativa afecta

Sistema 5. POLARIDAD 
En la lengua bribri, la polaridad negativa afecta formalmente al Finito:

(21)

\begin{tabular}{|l|l|l|l|l|l|}
\hline $\begin{array}{l}\text { be' } \\
2 S\end{array}$ & $\begin{array}{l}\text { Lè } \\
\text { tal vez }\end{array}$ & $\begin{array}{l}\text { ye' } \\
1 S\end{array}$ & $\begin{array}{l}\text { lè } \\
\text { tal vez }\end{array}$ & $\begin{array}{l}\text { kè kũ } \\
\text { NEG estemos }\end{array}$ & $\begin{array}{l}\text { iã } \\
\text { más }\end{array}$ \\
\hline Sujeto & $\begin{array}{l}\text { Adjunto } \\
\text { modo }\end{array}$ & Sujeto & $\begin{array}{l}\text { Adjunto } \\
\text { modo }\end{array}$ & Finito^Predicador & $\begin{array}{l}\text { Adjunto } \\
\text { modo }\end{array}$ \\
\hline \multicolumn{7}{|l|}{ Modo } & Residuo & Modo \\
\hline \multicolumn{7}{|l|}{ Tal vez usted y yo nos vamos a morir pronto (3:36) } \\
\hline
\end{tabular}

(22)

\begin{tabular}{|l|l|l|l|l|}
\hline $\begin{array}{l}\text { kề } \\
\text { NEG }\end{array}$ & $\begin{array}{l}\text { wồyök } \\
\text { mal-agüero }\end{array}$ & $\begin{array}{l}\text { sũwềkũ } \\
\text { no-vemos }\end{array}$ & $\begin{array}{l}\text { iã } \\
\text { más }\end{array}$ & $\begin{array}{l}\text { sö } \\
\text { 1PL.ERG }\end{array}$ \\
\hline Finito & Complemento & Predicador^Finito & Adjunto Modo & Sujeto \\
\hline Modo & Residuo & Modo \\
\hline
\end{tabular}

(23)

\begin{tabular}{|l|l|l|l|l|}
\hline $\begin{array}{l}\text { se' } \\
\text { 1PL }\end{array}$ & $\begin{array}{l}\text { kë́ } \\
\text { NEG }\end{array}$ & $\begin{array}{l}\tilde{A} \\
\text { Para }\end{array}$ & $\begin{array}{l}\text { i } \\
\text { eso }\end{array}$ & $\begin{array}{l}\text { yörpa } \\
\text { era-posible }\end{array}$ \\
\hline Suj- & Finito & -eto & Complemento & Predicador ${ }^{\wedge}$ Finito \\
\hline Modo & Residuo & Modo \\
\hline \multicolumn{4}{|l}{ Nosotros no hubiéramos podido eliminarlos (3:51) } \\
\hline
\end{tabular}

En (21), se puede observar como el auxiliar $t s o^{\prime}$ se reemplaza mediante la forma supletiva $k \tilde{u}$ cuando la cláusula es negativa. Paralelamente, en (22), el sufijo de imperfectivo habitual -ke cambia su forma a -kũ en la negación. En (23), -pa es la forma negativa del sufijo -mĩ, que expresa posibilidad. Como se establece en los ejemplos, la negación kë se analiza desde la perspectiva sistémico-funcional como parte de la estructura del Modo, pues la polaridad es una característica concomitante del elemento Finito y es, por ende, parte de este. Contrariamente, 'sí' o 'no' se consideran como un Adjunto de Modo, en caso de respuesta a una pregunta, reconocimiento de una declaración o acatación de una orden; o bien como un continuativo que indica un nuevo turno y, en este caso, forman parte del tema textual.

Cabe destacar que en bribri la polaridad marcada tiene, en algunos casos, una doble marcación: (i) la presencia de la negación kë̀ y (ii) un cambio formal en el verbo. El análisis sistémico funcional da cuenta de esta doble realización. En (23), la negación kë es parte del Finito y - $p a$ que constituye el elemento Finito expresa dos opciones de escogencia de distintos subsistemas del Sistema FINITO: a) la opción 'negativo' en el subsistema de POLARIDAD y b) la opción 'posibilidad' en la entrada de 'modalización' en el subsistema de TIPO DE MODALIDAD.

\subsubsection{La polaridad negativa en cláusulas perfectivas}

La polaridad en bribri influye en la transitividad de las cláusulas perfectivas negativas. Al respecto, Constenla Umaña et al. indican lo siguiente: 
Las formas activas improspectiva [...] y la prospectiva [...] sólo pueden emplearse en las oraciones afirmativas. Cuando la oración es negativa, hay que reemplazarlas por la forma improspectiva de voz media. [...] En consecuencia, la diferencia entre improspectivo y prospectivo se neutraliza con la negación. Si la oración es transitiva, en estos casos se emplea como marcador del tópico (el agente semántico o, en su defecto, el actante más capaz de realizar acciones) la posposición wã en vez de la posposición tö (o dör). Esto se debe a que las formas de voz media son, por definición no transitivas y wã es la posposición que se emplea con los agentes semánticos en bribri siempre que a una forma no transitiva se le da un uso transitivo. (1998: 52)

Margery Peña, sin embargo, sostiene que la posposición wã es un marcador de ergatividad:

\begin{abstract}
En el caso de los sujetos agentes de oraciones oblicuas, los marcadores de la ergatividad corresponden a las expresiones dör, tö tö y wã. [...] En la totalidad de las correspondientes formas negativas, así como en las afirmativas del antepresente remotospectivo el marcador de ergatividad es wã, tanto en Katsi como en Amubre y Salitre. (2005: 43)
\end{abstract}

En contraste con esta perspectiva, Jara Murillo (1995b: 98) afirma que en bribri el aspecto perfectivo y la transitividad no pueden codificarse simultáneamente en una cláusula cuando esta es negativa, lo que comprueba el hecho de que la transitividad prototípica es un fenómeno relacionado con hechos ocurridos, típicamente codificados en cláusulas realis afirmativas perfectivas. La autora compara las siguientes cláusulas:

(a) Kë̀ ye' wã i ñằnẽ. 'Yo no lo comí'

(b) Kë̀ye' dör i ñẽ̀. 'Yo no lo comía'

Tomando en cuenta los parámetros de transitividad prototípica de Hopper y Thompson (1980), entre los cuales están el aspecto perfectivo, la polaridad afirmativa y el modo realis, Jara Murillo sostiene que al comparar a y b: a es más alta en transitividad de acuerdo con el rasgo de aspecto, pues es una cláusula perfectiva; mientras que b es imperfectiva. Sin embargo, la autora afirma que a es también más baja en transitividad, puesto que el verbo se codifica obligatoriamente en voz media y que, por lo tanto, su agente no puede marcarse como ergativo sino como un caso oblicuo con la posposición wã.

Desde la perspectiva de la metafunción ideacional, se podría hipotetizar que wã marca un grado de agentividad menor que tö, puesto que la cláusula, siendo negativa y en pasado, es algo que nunca ocurrió, es decir, el agente nunca llevó a cabo el proceso ${ }^{8}$. Paralelamente, desde el punto de vista interpersonal, wã podría marcar la diferencia en el grado de responsabilidad modal del Sujeto. Esto es, cuando el Sujeto está marcado con wã es menos responsable, en el continuum de responsabilidad modal, que cuando aparece marcado con $t \ddot{o}$, en el caso de los verbos típicamente transitivos, como en (24):

\begin{tabular}{|c|c|c|c|c|}
\hline $\begin{array}{l}\text { kề } \\
\text { NEG }\end{array}$ & $\begin{array}{l}\text { ie' wã } \\
\text { 3S AG }\end{array}$ & $\begin{array}{l}\text { piña } \\
\text { piña }\end{array}$ & $\begin{array}{l}\text { iènềwã } \\
\text { puso }\end{array}$ & $\begin{array}{l}\text { i ã } \\
\text { eso en }\end{array}$ \\
\hline Finito & Sujeto & Complemento & Predicador^Finito $^{\wedge}$ & Adjunto \\
\hline \multicolumn{2}{|l|}{ Modo } & Residuo & Modo & Residuo \\
\hline \multicolumn{5}{|c|}{ No le puso piña $(2: 14)$} \\
\hline
\end{tabular}

En el ejemplo (25), sin embargo, no se puede afirmar que el Sujeto/Agente sea menos responsable modalmente que en (24): 
(25)

\begin{tabular}{|l|l|l|}
\hline $\begin{array}{l}\text { ye' wã } \\
1 \text { S AG }\end{array}$ & $\begin{array}{l}\text { I } \\
\text { eso }\end{array}$ & $\begin{array}{l}\text { dé } \\
\text { llegué }\end{array}$ \\
\hline Sujeto & Complemento & Predicador $^{\wedge}$ Finito \\
\hline Yo lo traje
\end{tabular}

En (25), se observa el uso de $w a \tilde{c}$ con un verbo de movimiento. Según Constenla Umaña et al. (1998), en este caso el $w \tilde{a}$ se utiliza como reemplazo de tö en oraciones en las que a formas verbales intrínsecamente intransitivas, que implicarían sólo la presencia de un absolutivo, se les da un uso transitivo. El verbo dök en bribri es en principio intransitivo y tiene el significado de llegar, pero cuando en la cláusula se incluye otro participante, como el Agente/Sujeto en (25) ye wã , el verbo adquiere el significado de traer y el Agente se marca por medio de wã. Puesto que el Agente/Sujeto en (23) no constituye un ejemplo de Agente prototípico de cláusulas transitivas y que el verbo es, en principio, intransitivo, en el caso de los verbos de movimiento la responsabilidad modal del Sujeto (participante añadido) no es menor o nula. En efecto, en (25), ye' es el responsable de la validez de la proposición.

Por otro lado, (24) es una cláusula indicativa por medio de la cual se negocia cierto tipo de información: en este caso el Sujeto es el elemento de significado que el hablante pone en riesgo, es decir, es el candidato con base en el cual el oyente puede rechazar la proposición. La marcación wã podría interpretarse de la siguiente manera: el hablante le indica al oyente que el Sujeto de la proposición no está en riesgo alguno en cuanto a su responsabilidad modal en la cláusula, puesto que lo que se afirma a través de ella, más bien se niega, es decir, nunca ha ocurrido. Ergo, el Sujeto tiene menos probabilidades de ser el elemento por el cual el oyente rechace la proposición, respecto a si estuviera marcado con tö y la cláusula fuera afirmativa.

Sin embargo, en los casos en los que el wã marca la presencia de un participante añadido en la cláusula, lo que determina este tipo de marcación no es un grado inferior o nulo de responsabilidad modal, sino el hecho de que el verbo de movimiento no es prototípicamente transitivo y que, por lo tanto el paciente no está siendo afectado. Esto se puede representar como sigue:

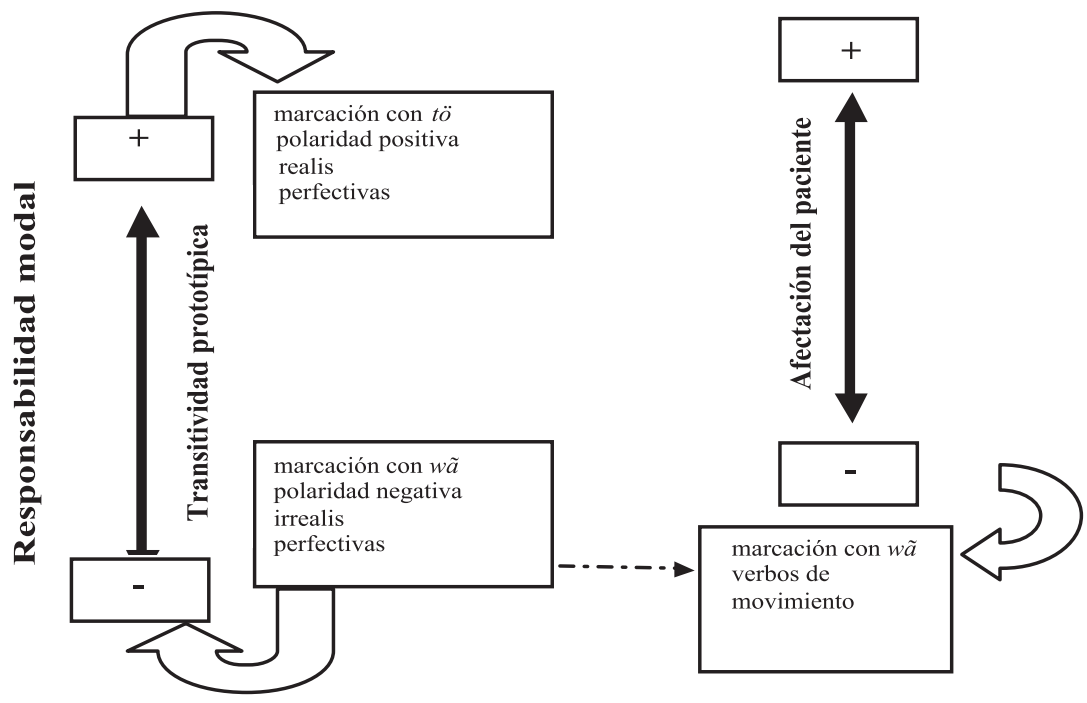

Figura 3. Relación entre responsabilidad modal, transitividad prototípica y afectación del paciente en la marcación con $w \tilde{a}$ 
En cuanto a los distintos grados de transitividad en la cláusula, según Givón (1984: 154), las cláusulas con sujetos menos agentivos son menos propensas a tener un sujeto ergativo. El patrón de marcación entre ergativo y no ergativo también puede darse en las lenguas ergativas bajo algunas condiciones de tiempo y/o aspecto como, por ejemplo, en bribri cuando la cláusula es perfectiva y negativa. De acuerdo con este autor, el grado de afectación del paciente puede crear variaciones en los patrones de ergativo en relación con el paradigma verbal: en este tipo de cláusulas el verbo aparece en efecto en voz media y la marcación de ergativo no se presenta.

En cuanto a lo que se refiere a la relación entre perfectividad y transitividad, Givón (1984: 156) afirma que en un evento perfectivo o completivo los límites de comienzo y conclusión están claramente especificados. Lo que es relevante es sobre todo la terminación del evento y el hecho de que haya ocurrido y se haya completado. La relación de la perfectividad con la transitividad se basa, por lo tanto, en dos inferencias pragmáticas: (i) afectación del paciente: cuanto más completo es el evento, es más probable que el paciente reciba los efectos de la acción; y (ii) efectividad del agente: cuanto más exitosamente se complete un evento, es más probable que el agente fuera en efecto la causa deliberada y directa de esa conclusión exitosa. Con base en estas consideraciones, la marcación con wã podría indicar también que la efectividad del agente es menor cuando la cláusula es perfectiva y negativa, puesto que el evento del que se habla nunca se ha completado, por lo cual la afectación del paciente será nula respecto de una cláusula perfectiva y afirmativa.

El hecho de que la polaridad negativa afecte la transitividad de la cláusula cuando esta es perfectiva ${ }^{12}$ se puede ejemplificar, desde la perspectiva sistémico-funcional, de la siguiente manera:

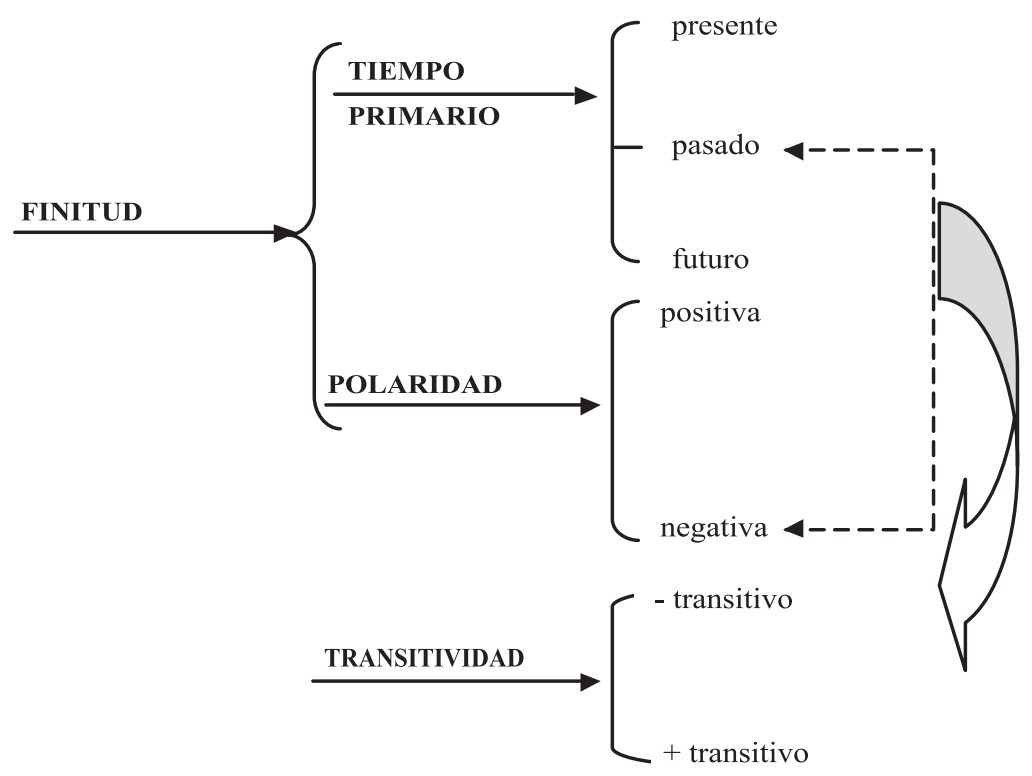

Sistema 6. TIEMPO PRIMARIO, POLARIDAD Y TRANSITIVIDAD

\subsection{El Sistema de FINITO}

El siguiente sistema describe todas las opciones del Sistema de FINITO analizadas por separado en los apartados anteriores: 


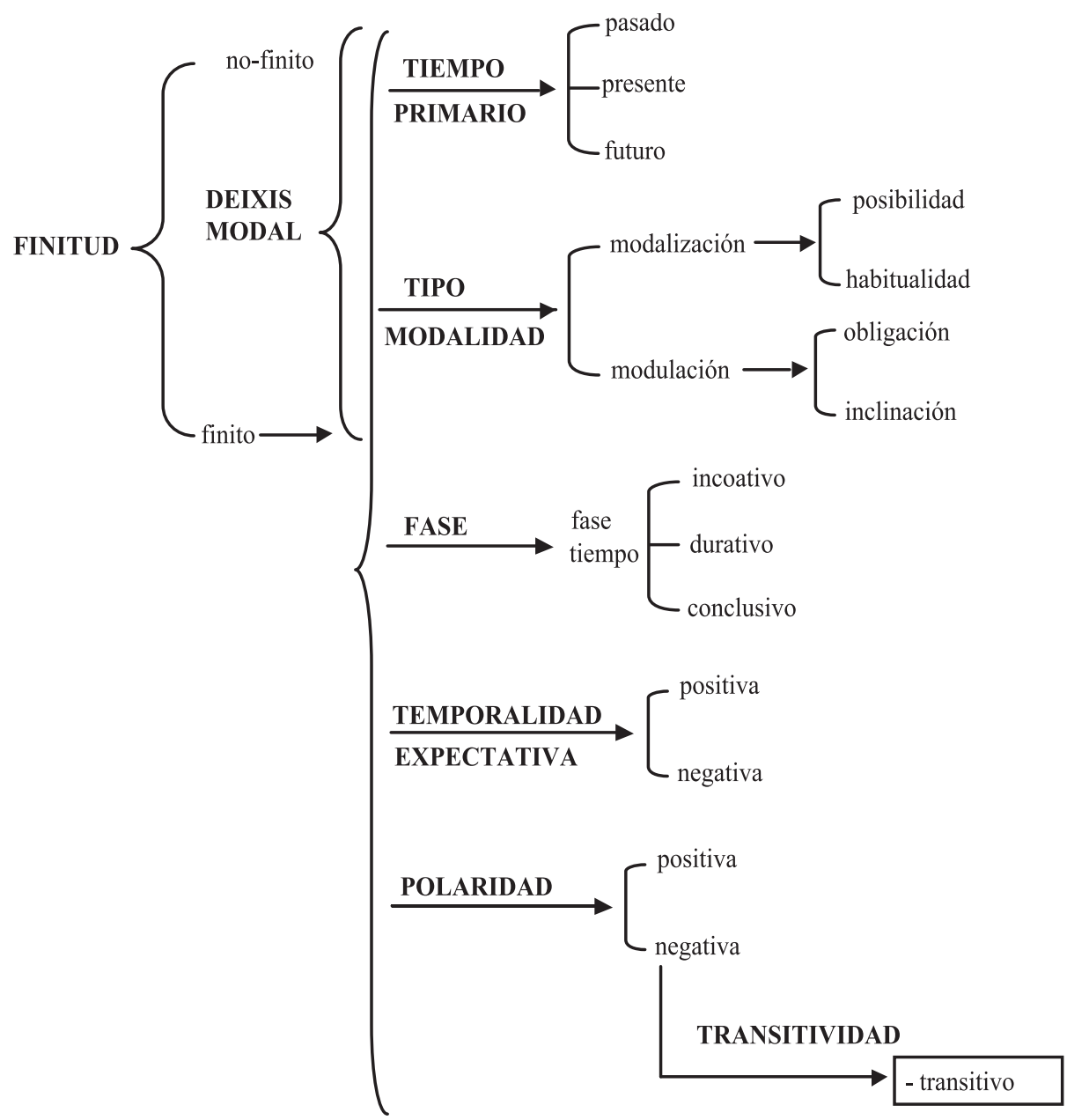

Sistema 7. FINITO

Como se puede observar en el sistema 7, la deixis de la cláusula se circunscribe en bribri por medio del elemento Finito, que tiene cinco posibles entradas: TIEMPO PRIMARIO (pasado, presente, futuro), MODALIDAD (probabilidad/habitualidad, inclinación/obligación), FASE DEL TIEMPO (incoativo, durativo, conclusivo), TEMPORALIDAD RELATIVA A LA EXPECTATIVA DEL HABLANTE (positiva/negativa) y POLARIDAD (positivo/negativo).

\subsubsection{La indeterminación en el Sistema de FINITO}

Halliday y Matthiessen (2006) sostienen que la indeterminación es una característica común y necesaria de un sistema semiótico en evolución. En cuanto al significado de indeterminación, los autores se plantean la siguiente pregunta:

What does it mean to say that a natural language is an indeterminate system? In most general terms, it suggests that the generalized categories that constitute language as a system -as "order" rather than as randomness or "chaos"- are typically not categorical: that is, they do not display determinate boundaries, fixed criteria of membership, or stable relationships from one stratum to another [...]. (Halliday y Matthiessen 2006: 547) 
Con respecto a la indeterminación en relación con la gramática, los autores esquematizan esta relación tomando en cuenta, entre otras, estas variables: a) dimensionalidad, y b) organización de categorías según el principio de indeterminación. La dimensionalidad describe el tipo de metafunción que se esté tomando en cuenta, es decir, ideacional (construcción de la realidad) o interpersonal (inteligencia social); mientras la organización se refiere a que las categorías de la lengua son difusas e, inclusive, redundantes.

La tarea de categorización equivale al manejo de la complejidad del lenguaje. Si un determinado fenómeno tiene que ser transformado en redes de significado potencial, esto implica que dicho fenómeno tiene que ser clasificado bajo múltiples perspectivas, con lo cual las áreas complejas de la experiencia tendrán múltiples ubicaciones para esto en el mapa gramatical. Los fenómenos de cualquier índole parecen significativamente diferentes si se analizan 'desde arriba' o 'desde abajo', como sucede en el caso del lenguaje. En el caso específico del fenómeno de la gramática, ésta tiene que lidiar entre las dos visiones y llegar a un punto de acuerdo. Cuando se trata de modelar las categorías escurridizas y naturalmente difusas de la experiencia humana, como el tiempo o la agentividad, la gramática recurre a varios tipos de indeterminación (Halliday y Matthiessen 2006: 549): (i) ambigüedades, (ii) mezclas, (iii) traslapes, (iv) neutralizaciones y (v) complementariedades:

Tabla 7. Tipos de indeterminación

\begin{tabular}{|l|l|}
\hline Tipos de indeterminación & Relación \\
\hline $\begin{array}{l}\text { Ambigüedad: } \\
\text { una determinada realización interpreta dos distintos } \\
\text { significados, cada uno de los cuales excluye al otro. }\end{array}$ & 'o $a$ o $x$ ' \\
\hline $\begin{array}{l}\text { Mezcla: } \\
\text { una determinada realización interpreta dos distintos } \\
\text { significados, y ambos se fusionan en uno. }\end{array}$ & 'tanto $b$ como $y$ ' \\
\hline $\begin{array}{l}\text { Traslape: } \\
\text { dos categorías se traslapan y ciertos miembros muestran } \\
\text { características de ambas. }\end{array}$ & 'parcialmente $c$, parcialmente $z '$ \\
\hline $\begin{array}{l}\text { Neutralización: } \\
\text { en ciertos contextos la diferencia entre dos categorías } \\
\text { desaparece. }\end{array}$ & - \\
\hline $\begin{array}{l}\text { Complementariedad: } \\
\text { algunas características o campos semánticos se construyen } \\
\text { de dos formas contradictorias. }\end{array}$ & - \\
\hline
\end{tabular}

En bribri, estos tipos de indeterminación se reflejan particularmente en las realizaciones sintagmáticas de los subsistemas del Sistema de FINITO. Tomando en cuenta el análisis realizado en este apartado, se pueden esbozar, para el Sistema de FINITO, algunos tipos de indeterminación.

La forma de imperfectivo simple en bribri constituye un ejemplo de traslape y, a la vez, de mezcla. El traslape del imperfectivo simple se debe al hecho de que este codifica tanto eventos presentes como eventos pasados en el subsistema de TIEMPO PRIMARIO, es decir, construye dos distintos significados. Además, es un ejemplo de mezcla, puesto que también es un recurso para la codificación de la habitualidad en el subsistema TIPO DE MODALIDAD y de durativo en el subsistema FASE DEL TIEMPO. En los ejemplos presentados en este 
apartado el límite entre habitualidad y tiempo durativo es difusa: un evento es habitual porque es algo que da repetidamente a través del tiempo, y uno de los recursos del bribri para expresar ambos conceptos es el imperfectivo simple.

Paralelamente, el sufijo - $m \tilde{\imath}$ puede codificar posibilidad, tiempo futuro y fase del tiempo incoativo. En la codificación de la posibilidad y tiempo futuro, el sufijo -mĩ puede considerarse como un ejemplo de neutralización, es decir, un tipo de indeterminación en el que la diferencia entre dos categorías desaparece. Además, si se considera que -mĩ puede expresar también fase del tiempo incoativo, este sufijo, aparte de ser ejemplo de neutralización en ciertos contextos, constituye también un caso de traslape, es decir, de la relación 'parcialmente $c$, parcialmente $z$ ': las categorías de futuro/posibilidad y fase del tiempo incoativo se traslapan en el sufijo - $m \tilde{\imath}$, que codifica ambos.

Otra instancia prototípica de la indeterminación en el Sistema de FINITO en bribri, es la forma de imperfectivo habitual, que puede codificar situaciones presentes y futuras en el subsistema de TIEMPO PRIMARIO, tiempo durativo en el subsistema FASE DEL TIEMPO y habitualidad en el subsistema de TIPO DE MODALIDAD. Al igual que el imperfectivo simple, en el caso de la codificación de tiempo durativo y habitualidad, el sufijo - $k e$ de imperfectivo habitual constituye otro ejemplo de mezcla y, a la vez, de traslape en la codificación de eventos tanto futuros como presentes.

\section{7. $\quad$ El Predicador}

Según Halliday (2004: 122), el Predicador tiene cuatro funciones básicas: (i) especificar el tiempo secundario relativo al tiempo primario, (ii) establecer aspectos y fases, (iii) indicar la voz y (iv) determinar el tipo de proceso que se predica del Sujeto. En bribri, sin embargo, parte de estas funciones del Predicador están realizadas en el Finito. Como se mencionó en el apartado 4.5.2, la determinación de fases y/o aspectos se realiza en bribri en el subsistema de FASE, que es parte del Sistema de FINITO. El tiempo secundario relativo al tiempo primario es parte, en el marco hallidiano, de la red de sistemas de organización lógica del grupo verbal y, por lo tanto, no se tomará en cuenta en esta investigación.

A raíz de estas consideraciones, en bribri el Predicador debería desempeñar, en principio, dos funciones: (i) determinar el tipo de proceso y sus incrementos, e (ii) indicar la voz. La determinación del tipo de proceso (acción, evento, proceso mental, relación) recae dentro de la metafunción ideacional, así como el incremento de los distintos procesos, que ha sido esbozado en la tabla 4.8. En cuanto a la voz, en bribri se realiza morfológicamente en el Finito, aunque está expresada semánticamente también en el Predicador, puesto que algunos verbos son intrínsecamente de voz media. Sin embargo, el Sistema de VOZ no ha sido incluido en el Sistema de FINITO en esta investigación, puesto que es parte, según la gramática sistémicofuncional, de la red de sistemas del grupo verbal (Halliday 2004: 349) y cuenta únicamente con dos posibles entradas, activa y pasiva, que no son relevantes en el caso del bribri.

\section{Conclusiones}

A partir del análisis propuesto por Halliday, la red de escogencias del Sistema MODO se realiza en la lengua en la estructura sintagmática del Modo, es decir, las distintas realizaciones de Sujeto^$\wedge$ Finito establecen las posibilidades de escogencia del hablante para poder realizar gramaticalmente funciones de habla tales como órdenes, declaraciones y preguntas. En bribri, el 
elemento que realiza las escogencias del Sistema TIPO DE MODO (declarativa, interrogativa, imperativa) es el Finito. En el caso de las cláusulas declarativas e imperativas, es la flexión verbal la que determina el MODO de la cláusula. En el caso de las interrogativas polares, el MODO se codifica por medio de la entonación. Marginalmente, se ha observado que, en los textos analizados, cuando la cláusula es imperativa y transitiva el Sujeto tiende a elidirse, esto debido al hecho de que los dos constituyentes fundamentales de la cláusula bribri son el objeto y el verbo.

La descripción del Sistema de MODO ha conllevado el análisis de los subsistemas que caracterizan la cláusula como intercambio dialógico: MODALIDAD y FINITO. El Sistema de MODALIDAD en bribri cuenta con cuatro posibles escalas de modalidad: inclinación y obligación (modulación), y posibilidad y habitualidad (modalización). En los textos, se ha determinado que las escalas de modalidad pueden tener realizaciones congruentes, analizables en el marco de la metafunción interpersonal, y metafóricas, pertenecientes al dominio de la metafunción ideacional. Las realizaciones congruentes se realizan en la lengua por medio del elemento Finito. La complejidad y multiplicidad de funciones del elemento Finito se refleja en el hecho de que este realiza, por lo menos, dos subsistemas de manera simultánea en la cláusula bribri.

Halliday (2004: 115) indica que el Finito circunscribe la deixis de la cláusula expresando (i) tiempo primario, (ii) modalidad y (iii) polaridad. El Sistema de FINITO en bribri es sumamente complejo. Además de circunscribir la deixis de la cláusula temporalmente por medio del subsistema de TIEMPO PRIMARIO, modalmente por medio del Sistema de MODALIDAD o expresando una de las dos opciones del Sistema de POLARIDAD, el Finito en bribri también engloba los siguientes subsistemas: TEMPORALIDAD RELATIVA A LA EXPECTATIVA y FASE DEL TIEMPO.

El Sistema de FASE es incluido por Halliday (2004: 520) en el análisis de los procesos de expansión del grupo verbal y consta de dos posibles entradas: fase del tiempo y fase de la realidad. La entrada fase del tiempo cuenta con tres posibles opciones de escogencia: incoativo, durativo y conclusivo. La entrada fase de la realidad, por otro lado, se basa en el contraste entre 'aparente' ('parece ser') y 'realizado' ('resulta ser'). Debido a la imposibilidad de aplicar este contraste al análisis del Finito en bribri, el Sistema de FASE cuenta exclusivamente con la entrada 'fase del tiempo'. La entrada 'fase de la realidad' está estrechamente relacionada con la interpretación de los aspectos perfectivo e imperfectivo en la teoría sistémico-funcional:

\footnotetext{
The imperfective represents the real, or actual, mode of non-finiteness ('realis'), while the perfective represents the potential, or virtual ('irrealis'). [...] historically the imperfective combined with the preposition 'at, in'; the perfective combined - and still does, in the infinitive form- with the preposition 'to'. The meaning of the two aspects is very fluid and indeterminate; in the most general terms, the imperfective means act in progress, actual, present, ongoing, steady state or (dependent) proposition, while the perfective means goal to be attained, potential, future, starting and stopping, change of state or (dependent) proposal. (Halliday 2004: 425)
}

Considerando la 'fase de la realidad' a raíz de la distinción entre imperfectivo y perfectivo, el autor indica que:

\footnotetext{
the reality-phase, or realization, system is based on the contrast between 'apparent' (seems to be) and 'realized' (turns out to be); both are perfective, the first being unreal, the second unreal emerging into real. (Halliday 2004: 499)
}

Esta interpretación de perfectivo e imperfectivo en relación con el modo realis o irrealis ha sido limitante en el caso del bribri, puesto que ninguno de estos aspectos de la lengua se ha podido analizar a partir de la perspectiva sistémico-funcional. Constenla Umaña et al. sostienen que: 
La categoría más básica de la flexión verbal bribri es la voz, activa o media [...]. Le sigue en importancia el modo: indicativo, imperativo, optativo, etc. En el modo indicativo, se presentan oposiciones por las categorías de aspecto y tiempo, de las cuales, la fundamental es la primera. [...] La diferencia aspectual fundamental radica en la concepción de los acontecimientos como completos (aspecto perfectivo) o incompletos (aspecto imperfectivo). (1998: 15)

Debido a la interpretación antagónica de imperfectivo y perfectivo en la teoría sistémico-funcional respecto a la tradicional, no se han tomado en cuenta estos aspectos ni los modos realis e irrealis en el análisis textual. Este puede considerarse como un límite de la teoría en su aplicación específica a una lengua como el bribri.

Analizando el Sistema de FINITO en bribri se ha podido determinar que las realizaciones de los subsistemas que lo componen son fundamentalmente indeterminadas, con lo cual el límite entre estos es intrínsecamente difuso. En efecto, una misma realización puede codificar una o más entradas de distintos subsistemas, así como varias realizaciones pueden codificar una misma entrada de un determinado sistema o subsistema.

\section{Notas}

1 Los textos que se analizarán en el presente artículo son: "El mal agüero del gallo", un texto narrado por el awá Francisco García que forma parte de I ttè. Historias bribris (Jara Murillo: 1993); "Sĩõ'tãmĩ" y "Óköm", dos fragmentos de entrevistas extraídas de Cargos tradicionales del pueblo bribri: Sĩō'tãmĩ - Óköm - Awá (Jara Murillo y García Segura 2008); "Cómo se vota” (Wềs yểjkuö tawềke?), un texto instructivo traducido por Alí García Segura y una conversación informal entre Alí García Segura y su hermano Bahil García Segura, transcrita y analizada por Jara Murillo (1998 y 2004b).

2 La distinción entre Modo y MODO tiene valor teórico. Con 'Modo' se entiende la estructura sintagmática interpersonal de la cláusula (Modo + Residuo). 'MODO', por otro lado, se refiere al sistema paradigmático interpersonal de la cláusula, es decir, la gramaticalización del sistema semántico de función de habla en el que se abre una red de escogencias (declarativa/imperativa/interrogativa/afirmativa/exclamativa).

3. El primer número entre paréntesis, precedido de una $\mathrm{T}$, se refiere al texto del que fue extraída la cláusula. La numeración de los cinco textos será la siguiente:

T1: Cómo se vota.

T2: Conversación informal.

T3: El mal agüero del gallo.

T4: Fragmento de la Entrevista a la Siõ'tãmĩ Anastasia Segura.

T5: Fragmento de la Entrevista al Óköm Silverio Morales.

El segundo número que aparece, después de dos puntos, indica el número de cláusula de cada texto. Para la división de los textos en cláusulas véase Pacchiarotti (2009).

4. Las partes del cuerpo en bribri son de posesión inalienable, con lo cual es imprescindible especificar el poseedor.

5. Cf. Constenla Umaña et al. (1998: 112) y Jara Murillo (1995c: 11).

6. Cf. Constenla Umaña et al. (1998: 47).

7. Jara Murillo y García Segura (2009).

8. En el ámbito de esta investigación no se tomarán en cuenta, en la descripción del sistema verbal, las clases de verbos. Para un análisis del sistema verbal en relación con las clases de verbos cf. Jara Murillo (1995c).

9. Acerca de la formación del perfectivo e imperfectivo de voz media, cf. Constenla Umaña et al. (1998: 26 y 83) y Jara Murillo y García Segura (2009: 135 y 136).

10. Acerca de la formación del perfecto remoto e imperfectivo simple, cf. Constenla Umaña et al. (1998: 15 y 82) y Jara Murillo y García Segura (2009: 53 y 131).

11. Cf. Jara Murillo (1995 a) y Constenla Umaña et al. (1998: 52). 
12. En la perspectiva sistémico-funcional, los términos 'perfectivo' e 'imperfectivo' tienen un significado totalmente distinto al que normalmente se les atribuye en otras teorías gramaticales. Por esta razón se prefiere evitar el uso de 'perfectivo' y sustituirlo por 'pasado', en el sentido de algo concluido; en oposición a 'durativo', opción de escogencia del subsistema FASE DEL TIEMPO. Esta sustitución se justifica por el diferente significado que se le atribuye al término 'perfectivo' en la teoría sistémico-funcional: Halliday (2004: 425) considera que el perfectivo representa lo potencial o virtual es decir lo 'irrealis'.

\section{Bibliografía}

Constenla Umaña, Adolfo. 1991. Las lenguas del Área Intermedia: introducción a su estudio areal. San José: Editorial de la Universidad de Costa Rica.

Constenla Umaña, Adolfo et al. 1998. Curso Básico de Bribri. San José: Editorial de la Universidad de Costa Rica.

Givón, Talmy. 1984. Syntax. Vol. I. Amsterdam \& Filadelfia: Benjamins.

Halliday, Michael. 2004. An Introduction to Functional Grammar. London: Hodder Arnold.

Halliday, Michael y Christian Matthiessen. 2006. Construing experience through meaning: a language based approach to cognition. London: Continuum.

Hopper, Paul y Sandra Thompson. 1980. "Transitivity in Grammar and Discourse”. Language. 56 (2): 251-299.

Jara Murillo, Carla Victoria. 1993. I ttè. Historias Bribris. San José: Editorial de la Universidad de Costa Rica.

1995a. Text and context of the Sũwõ': Bribri Oral Tradition. PhD dissertation: Louisiana State University.

1995b. “Transitividad en el discurso bribri”. Revista de Filología y Lingüística. 21 (2): 93-105.

1995c. "Caracterización del sistema verbal bribri a partir del discurso narrativo de Coroma”. Estudios de lingüística Chibcha. 14: 7-29.

1998. "Estructura de la conversación y diálogo de géneros en la lengua bribri (chibcha)". Revista de Filología y Lingüística. 36 (1): 233-249.

2004a. "Observaciones para el estudio dialectológico de la lengua bribri". Estudios de Lingüística Chibcha. 23: 89-120.

2004b. "Hispanismos en la conversación bribri (familia chibcha)". Memoria del XIII Congreso internacional de la Asociación de Lingüística y Filología de América Latina (ALFAL). Universidad de Costa Rica.

Jara Murillo, Carla Victoria y Alí García Segura. 2008. Cargos tradicionales del pueblo bribri: Sĩơ'tãmĩ-Óköm - Awá. San José: Instituto Costarricense de Electricidad.

2009. Se' é ' yawö bribri wa. Aprendemos la lengua bribri. UCR-UNICEF.

Margery Peña, Enrique. 2005. Diccionario fraseológico Bribri-Español, Español-Bribri. San José: Editorial de la Universidad de Costa Rica.

Pacchiarotti, Sara. 2009. La cláusula como intercambio en bribri según la gramática sistémico-funcional. Tesis de Maestría en Lingüística: Universidad de Costa Rica. 